\title{
Differential methylation hybridization profiling identifies involvement of STAT1-mediated pathways in breast cancer
}

\author{
JU HEE KIM ${ }^{1}$, HAN-SUNG KANG ${ }^{2}$, TAE WOO KIM ${ }^{1}$ and SUN JUNG KIM ${ }^{1}$ \\ ${ }^{1}$ Department of Life Science, Dongguk University-Seoul, Seoul 100-715; ${ }^{2}$ Research Institute \\ and Hospital, National Cancer Center, Gyeonggi do 411-764, Republic of Korea
}

Received April 12, 2011; Accepted May 26, 2011

DOI: 10.3892/ijo.2011.1075

\begin{abstract}
Many cancer-related genes are regulated by an epigenetic mechanism through modification of the methylation status of $\mathrm{CpG}$ sites at the promoter. This study was carried out at a genome-wide scale to mine genes in which the methylation of $\mathrm{CpG}$ sites is altered in breast cancer tissues. Differential methylation hybridization analysis was conducted using a chromosomal DNA mixture of ten normal and cancer tissue sets. A CpG microarray harboring 237,220 CpG sites of the whole genome was interrogated and the resulting methylation level differences, as well as the RNA expression differences, between the normal and cancer sets for selected genes were verified in breast cell lines by methylation-specific PCR and real-time PCR analyses. As a result, we identified and verified novel genes that were hypermethylated in breast cancer, such as NRN1, CA5B and RPIA. Pathway analysis of the genes with altered methylation patterns identified the involvement of a differentiation-related network of genes whose activity may be heavily regulated by STAT1 in breast tumorigenesis. Our results suggest that epigenetic dysregulation of cellular processes relevant to STAT1-dependent cellular differentiation may be intimately involved in breast carcinogenesis. These findings lend credence to the possibility of using tumor-specific alterations in methylation patterns as biomarkers in estimating prognosis and assessing treatment options for breast cancer.
\end{abstract}

Correspondence to: Dr Sun Jung Kim, Department of Life Science, Dongguk University, Seoul 100-715, Republic of Korea

E-mail: sunjungk@dongguk.edu

Dr Han-Sung Kang, Research Institute and Hospital, National Cancer Center, Gyeonggi do 411-764, Republic of Korea

E-mail: rorerr@ncc.re.kr

Abbreviations: $\mathrm{CpG}$, cytosine guanine dinucleotide; DMH, differential methylation hybridization; MSP, methylation-specific PCR; RT-PCR, reverse transcription-polymerase chain reaction

Key words: breast cancer, $\mathrm{CpG}$ island, differential methylation hybridization, methylation, STAT1

\section{Introduction}

Epigenetic regulation of gene expression via $\mathrm{CpG}$ methylation plays a key role in cell growth, differentiation, apoptosis and tumorigenesis $(1,2)$. CpG sites at promoters have been intensively studied because their hypermethylation is associated with inactivation of tumor suppressor and thereby prompts carcinogenesis (3). Upon methylation of the $\mathrm{CpG}$ site, transcriptional activators cannot access their target sites and/or the DNA structure can be modified by altering the chromatin structure (4). So far, many genes have been identified as undergoing methylation changes during tumorigenesis in many types of cancer. Wellknown tumor suppressors in this category in breast cancer are RASSF1A, BRCA1, PCDH8, and 15-Hydroxyprostaglandin (5-8). Proto-oncogenes, on the other hand, undergo hypomethylation to contribute to carcinogenesis. FEN1 and IGF2 $(9,10)$ are representative oncogene markers in breast cancer.

The methylation markers have been mined at the level of individual genes, as well as genome-wide. Mining at the gene level was carried out by monitoring the methylation of $\mathrm{CpG}$ sites using bisulfate sequencing or methylation-specific PCR for individual tumor suppressors or oncogenes (11). Genome-wide scanning of $\mathrm{CpG}$ sites in breast cancer has been performed in just a few cases. Yan et al developed and performed an array-based method, called differential methylation hybridization (DMH), in breast cancer cell lines and in cancer tissues $(12,13)$. They used an array panel containing $1104 \mathrm{CpG}$ island tags. This technique was improved as the number of $\mathrm{CpG}$ tags were increased to 28,000 , and HOXB13 and HNF1B were identified as novel breast cancer markers (14). More recently, a genotyping-based technique called the Illumina methylation array was developed that covers 27,578 or $450,000 \mathrm{CpG}$ sites. Using this method, a methylation profile was obtained according to estrogen and progesterone receptor status in breast cancer (15).

The major advantage of genome-wide screening over individual screening is that the former can provide systemic information of the $\mathrm{CpG}$ sites scattered over the whole genome. To understand the biologically relevant pathways responsible for carcinogenesis, systemic approaches are essential. Even though genome-wide screening has been carried out in breast cancer in a few cases, the pathway analysis is rarely available. Only Inflammatory Response and Connective Tissue Disorders pathway is reported in the case of estrogen receptor-positive breast cancer (15). 
In this study, we performed a differential methylation hybridization assay covering $237,220 \mathrm{CpG}$ sites to identify genes that are differentially methylated in breast cancer using breast tumor and normal tissues. Methylation-specific PCR and real-time quantitative RT-PCR analyses were performed to verify the methylation data. Identified genes with differential methylation patterns in tumor versus normal tissue were further analyzed using bioinformatic tools to detect cancer-related pathways that may play a role in breast tumorigenesis.

\section{Materials and methods}

Genomic DNA extraction from breast tissues. Post-surgical samples of breast carcinoma and nearby normal tissues were obtained from National Cancer Center in Goyang, Korea. The patients' informed consent for participation in the study was obtained. As chemo- and radiotherapy have previously been implicated in the alteration of methylation patterns, no subjects who had received either type of treatment were included in the study. A few milligrams of tumor and adjacent normal tissue were taken and chromosomal DNA was isolated as described previously (11).

Immunoprecipitation and amplification of methylated DNA. Two micrograms of each cancer tissue DNA from 10 cancer patients were mixed together and the DNA was fragmented into 300-500 bp using a sonicator (Bioruptor, model; UCD200TM-EX) at low power for $10 \mathrm{~min}$ with repeating cycles of $15 \mathrm{sec}$ on and $15 \mathrm{sec}$ off. The normal tissue DNA was also processed in parallel with the cancer tissue DNA. DNA containing methylated $\mathrm{CpG}$ was immunoprecipitated using the MeDIP kit (Diagenode) according to the supplier's protocol, with final elution of $25 \mu \mathrm{l}$. Ten microliters of the eluted DNA was used for whole genome amplification (WGA) using the WGA kit (Sigma) according to the supplier's protocol. The amplified PCR product was purified using the PCR Purification kit (Bioneer, Korea).

Cell culture. Normal human breast cell lines, MCF-10A and MCF-12A, and cancer cell lines, MCF-7, MDA-MB-231, MDA-MB-468, SK-BR-3, T47D, and ZR-75-1, were purchased from the American Type Culture Collection (ATCC; Manassas, VA) and grown in Dulbecco's modified Eagle's medium (DMEM) supplemented with 10\% fetal bovine serum (FBS). To induce demethylation of the cytosine residues, a methyltransferase inhibitor, 5-Aza-2'-deoxycytidine, was added to the culture medium at $5 \mu \mathrm{M}$ for $72 \mathrm{~h}$. Culture medium with or without treatment was changed every $24 \mathrm{~h}$.

Differential methylation hybridization. For the whole-genomeamplified DNA, the human CpG Island Microarray (244K, Agilent) analysis was performed by Ebiogen (Korea). The microarray covers 27,800 CpG islands and contains 237,220 probes. Differential expression values, calculated as $+\mathrm{Cy} 3 / \mathrm{Cy} 5$ where $\mathrm{Cy} 3$ signal $>\mathrm{Cy} 5$ signal or $-\mathrm{Cy} 5 / \mathrm{Cy} 3$ where $\mathrm{Cy} 3$ signal $<$ Cy5 signal, were compared between the duplicate experiments. Clones differentially regulated in both experiments that had a significant ratio of Cy3 to Cy5 (defined as a value greater than 2) were selected and further analyzed.
Pathway analysis. To identify pathways displaying tumorspecific altered methylation patterns with potential roles in breast carcinogenesis, functional categorization and pathway construction were performed using the Ingenuity Pathway Analysis (IPA) software tool produced by Ingenuity Systems. IPA utilizes an extensive database of functional interactions that are drawn from peer-reviewed publications and are manually maintained (16). P-values for individual networks were obtained by comparing the likelihood of obtaining the same number of transcripts or greater in a random gene set as were actually present in the input file (i.e., the set of genes differentially methylated in normal and tumor tissue) using Fischer's exact test, based on the hypergeometric distribution. The highest confidence functional network was designated as the top network.

Methylation-specific PCR (MSP). Chromosomal DNA was isolated from the cell cultures in a $75-\mathrm{cm}^{2}$ culture flask using a genomic DNA purification kit (Promega, Madison, WI) according to the manufacturer's protocol. The extracted DNA was eluted with $250 \mu \mathrm{l}$ of distilled water. Sodium bisulfite modification of genomic DNA was carried out using an EpiTect Bisulfite kit (Qiagen, Valencia, CA) according to the manufacturer's protocol using $0.1 \mathrm{mg}$ of purified DNA. PCR was carried out using primers (Table I) and a Kapa SYBR Fast qPCR kit (Kapa Biosystems, Woburn, MA). A methylation index was calculated for each sample using the following formula: methylation index $=\left[1 /\left(1+2^{-(\mathrm{CTu}-\mathrm{CTme})}\right] \times 100 \%\right.$, as previously described (17), where CTu is the average cycle threshold (CT) obtained from duplicate quantitative PCR analyses using the unmethylated primer pair and CTme is the average CT obtained using the methylated primer pair.

Real-time RT-PCR. Total RNA from cell culture was prepared using Trizol reagent according to the manufacturer's protocols (Gibco-BRL, Carlsbad, CA). Reverse transcription was conducted using $10 \mu \mathrm{g}$ of total RNA with a reverse transcription kit (Promega). Expression levels of selected genes were measured by real-time quantitative RT-PCR analysis in order to confirm consistency with the microarray data. One microliter of cDNA was used for the PCR, and duplicate reactions were performed for each sample using a Kapa SYBR Fast qPCR kit (Kapa Biosystems) with gene-specific primers on an ABI 7300 instrument (Applied Biosystems). The primers for these selected genes are listed in Table I. RNA quantity was normalized to GAPDH content, and gene expression was quantified according to the $2^{-\Delta \mathrm{Ct}}$ method.

Statistical analysis. Genes from the microarray data that satisfied the following criteria were selected and submitted to IPA analysis: hypermethylated or hypomethylated in cancer tissue by more than two-fold; signal intensity higher than 1,000; $\mathrm{CpG}$ positioned within $-2,000$ to +500 . Student's t-test was used to detect differences in the mean values of the variables. $\mathrm{P}<0.05$ were considered statistically significant. All calculations were performed using SPSS for Windows, release 17.0 (SPSS Inc., Chicago, IL).

\section{Results}

Genome-wide differential methylation hybridization analysis of breast cancer. To identify genes that are regulated by gene 
Table I. Sequences of primers employed in this study.

\begin{tabular}{lll}
\hline Genes & \multicolumn{1}{c}{ Forward primer $\left(5^{\prime}-3^{\prime}\right)$} & \multicolumn{1}{c}{ Reverse primer $\left(5^{\prime}-3^{\prime}\right)$} \\
\hline MSP & & \\
NRN1-M & GTTTGTTTTGGAAATTTTTAAAGC & CCGAAAATTTAATTTACACACGTA \\
NRN1-U & TTGTTTTGGAATTTTTAAAGTGT & CCAAAAATTAATTTACACACATA \\
CA5B-M & GATTTTCGTTTTGGCGTATC & AAAACCTTAAAAAATAACAACCGTT \\
CA5B-U & GATTTTTGTTTTGTGTATTGG & AAAACCTTAAAAAATAACAACCATT \\
RPIA-M & TTTCGGGTTTTTAGTATTTTTAC & CACGAATCCCAAACTATACACG \\
RPIA-U & TTTTGGGTTTTTAGTATTTTTTATGG \\
RT-PCR & & CACAAATCCCAAACTATACACACC \\
NRN1 & & \\
CA5B & AGAGTCCACGCGTATCTGGT & CCAGTATGTGCACACGGTCT \\
RPIA & AAATCAGTGATCAAGGGAGGA & ATTTGCTGTCCACGGTGTG \\
STAT1 & GCGAATAGCTGAAAGGGTGA & TCCAGATCACTGAGGGTCAA \\
TMF1 & GAAAAGCAAGACTGGGAGCA & TTATCCTGAAGATTACGCTTGC \\
\end{tabular}

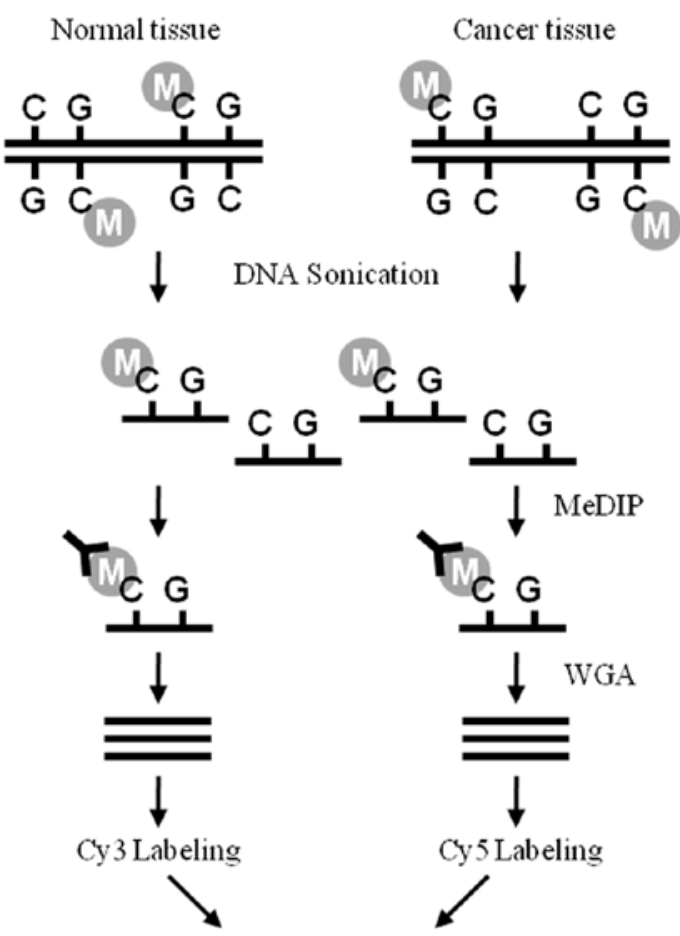

Hybridization and Microarray

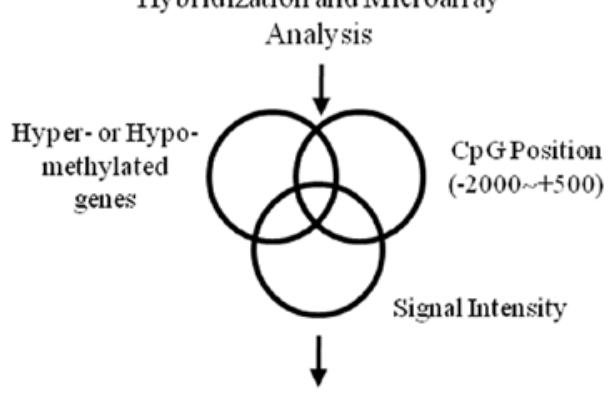

IPA Analysis

Figure 1. Experimental scheme of differential methylation hybridization of breast tissues. Chromosomal DNA was fragmented by sonication and subjected to immunoprecipitation using anti-CpG antibody. The DNA was then used for whole genome amplification and the amplified PCR amplicons were applied to $\mathrm{CpG}$ microarray. Hypermethylated and hypomethylated genes were pooled and pathway analysis was performed.

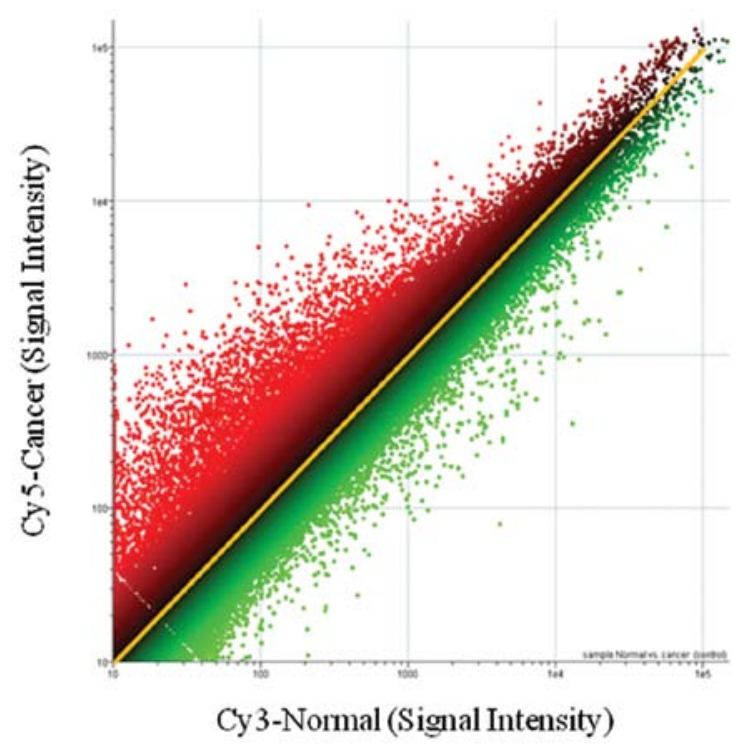

Figure 2. Genome-wide hypermethylation pattern in breast cancer. Methylation histogram of normal (X-axis) vs. cancer (Y-axis). The methylation level of 237,220 CpG sites was measured by differential methylation hybridization assay and is presented as a value of signal intensity: Cy5, tumor; $\mathrm{Cy} 3$, normal. The best fit line was added.

methylation and to further elucidate the regulatory pathways involved in breast carcinogenesis, differential methylation hybridization analysis for the whole genome $\mathrm{CpG}$ islands was carried out (Fig. 1). Of the 237,220 CpG sites, we found $>2$-fold signal in 13,407 hypermethylated sites, and <2-fold in 5,815 hypomethylated sites. We further screened the subgrouped genes according to their signal intensity in the hybridization. Genes showing intensity of $>1,000$ were selected from the normal tissue in the case of hypermethylation and from the cancer tissue in the case of hypomethylation. These selections included genes representing methylation change as well as high signal intensity. Finally, a pool of genes harboring $\mathrm{CpGs}$ within $-2,000$ to +500 was selected and contained 414 genes. The genome-wide methylation analysis found hypermethylation in $972 \mathrm{CpG}$ sites and hypomethylation in $209 \mathrm{CpG}$ sites, 
Table II. Representative 10 genes identified in this study by differential methylation hybridization and previously known to be hyper- or hypomethylated in breast cancer.

\begin{tabular}{llrlrr}
\hline Gene symbol & Genebank ID & Fold change & \multicolumn{1}{c}{ Gene description } & CpG site & eNorthern $^{\mathrm{b}}$ \\
\hline PCDH10 & NM_020815 & +77.99 & Protocadherin 10 & -746 & $0 / 0$ \\
MYO3A & NM_017433 & +6.52 & Myosin IIIA & +628 & $2 / 0$ \\
HIST1H2BK & NM_080593 & +3.08 & Histone cluster 1, H2bk & -163 & $6 / 0$ \\
FGF12 & NM_021032 & +3.0 & Fibroblast growth factor 12 & -189 & $0 / 0$ \\
PENK & NM_006211 & +15.6 & Proenkephalin & -314 & $1 / 0$ \\
ASCC3 & NM_022091 & -16.7 & Activating signal cointegrator 1 complex subunit 3 & +62 & $2 / 7$ \\
HSPA4 & NM_002154 & -10.0 & Heat shock 70 kDa protein 4 & +370 & $20 / 4$ \\
NDRG3 & NM_022477 & -10.0 & NDRG family member 3 & +415 & $14 / 1$ \\
BCAS3 & NM_017679 & -6.7 & Breast carcinoma amplified sequence 3 & -156 & $9 / 3$ \\
KIAA1524 & NM_020890 & -16.7 & KIAA1524 & -245 & $6 / 6$ \\
\hline
\end{tabular}

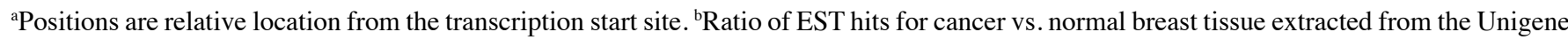
database.

corresponding to 1043 unique genes. These results suggest that the number of genes epigenetically inactivated in breast tumorigenesis greatly outnumbers the number of epigenetically activated genes (Fig. 2). Moreover, hypermethylation seemed to be a general pattern appearing across entire chromosomes, with no discernible differences in pericentromeric or subtelomeric regions (data not shown).

Genes previously identified as methylated in breast cancer such as PCDH10 (1.04-77.99-fold), MYO3A (0.79-6.52-fold), and HIST1H2BK (1.86-3.08-fold) also showed hypermethylation at the -1000 to +700 region (Table II). In addition, genes previously identified as hypomethylated in breast cancer such as HSPA4 (0.10-1.85-fold), NDRG3 (0.10-1.09-fold), and BCAS3 (0.16-3.44-fold) also showed hypomethylation in the same region. The results for these representative known genes support our methylation analysis. The consistency of our array data was also monitored by examining the methylation status of three randomly selected genes (NRN1, CA5B and RPIA) in cultured breast cell lines. Real-time methylation-specific PCR (MSP) and RT-PCR revealed hypermethylation and downregulation of all three genes in cancer cell lines, confirming the array data (Figs. 3 and 4). The involvement of methylation in the expression was examined by inducing demethylation of the hypermethylated $\mathrm{CpGs}$ with the methyltransferase inhibitor 5-Aza-2'-deoxycytidine. MSP and RT-PCR indicated that NRN1 gene expression was recovered upon demethylation (Fig. 5).

In addition to the known genes, novel genes for which the methylation status was not known were identified (Table III and IV). These included NXPH1, RAB12 and CDK7 for hypermethylation and ASCC3, HSPA4, and NDRG3 for hypomethylation.

Pathway analysis of the differentially methylated genes. All 1181 sites fitting our significance criteria for differential methylation were examined for functional interrelatedness using the Ingenuity Pathway Analysis software tool. The highest functional network (score $=59$ ) resulting from differential
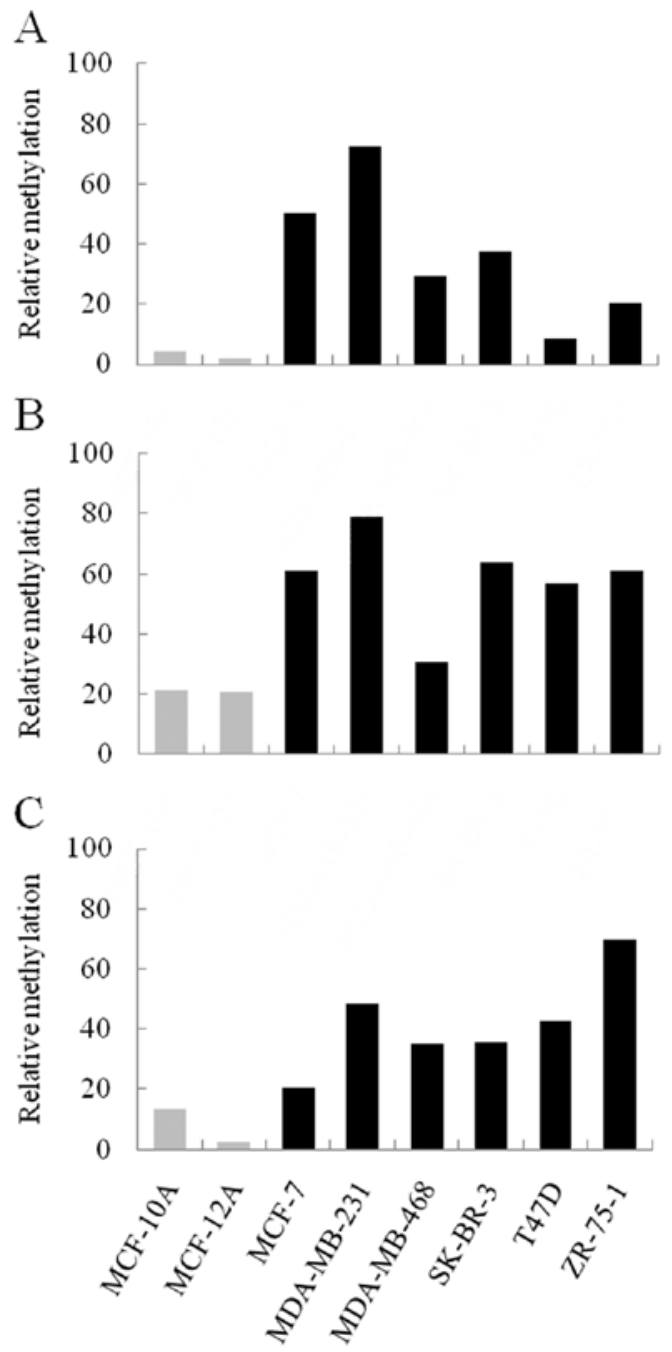

Figure 3. Analysis of methylation of the $\mathrm{CpG}$ sites in breast cancer cell lines. CpG sites located in NRN1 (A), CA5B (B), RPIA (C) that showed hypermethylation in the cancer tissue were randomly selected from the DMH data, and confirmed by real-time methylation-specific PCR in normal breast (gray bars) and breast cancer (black bars) cell lines. Each sample was examined in duplicate and the average relative methylation level is presented. 
A

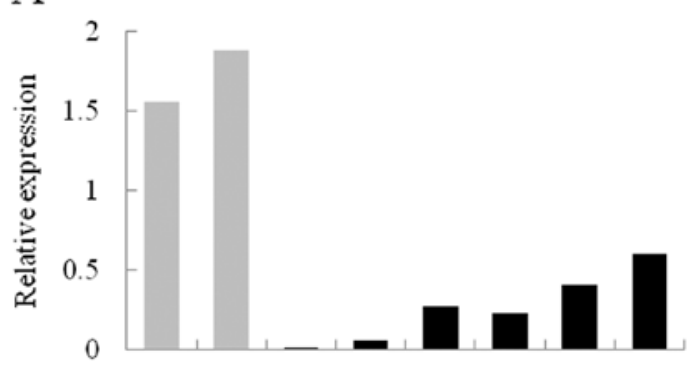

$\mathrm{B}$

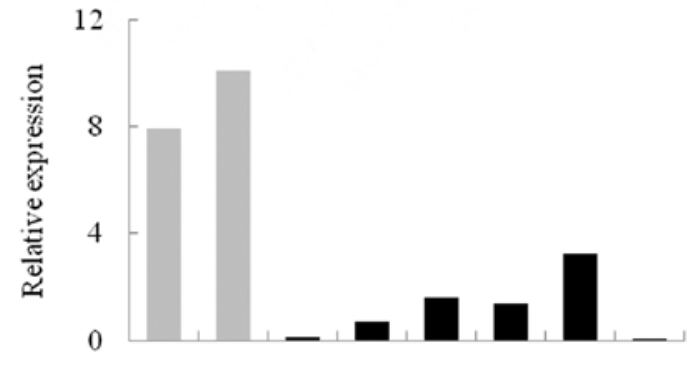

$\mathrm{C}$

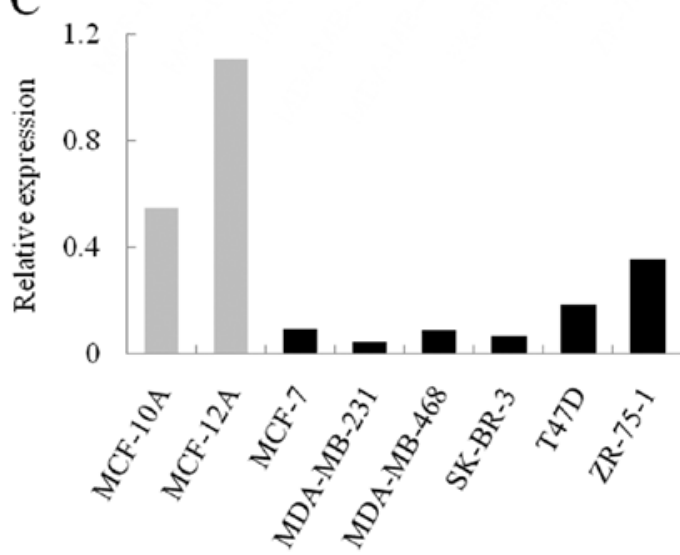

$\mathrm{D}$

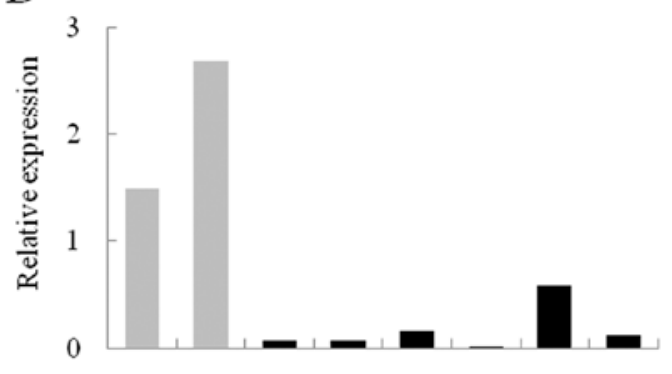

E

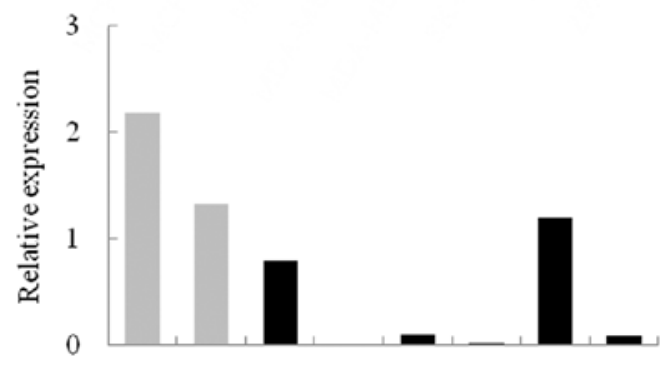

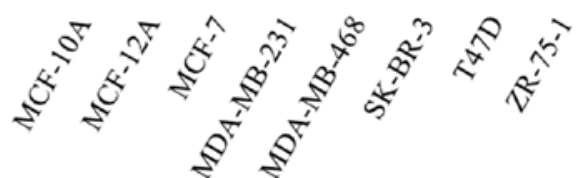

Figure 4. Real-time RT-PCR analysis of hypermethylated genes in breast cancer cell lines. Expression of genes [NRN1 (A), CA5B (B), RPIA (C), STAT1 (D) and TMF-1 (E)] that are hypermethylated in breast tumor was examined by real-time RT-PCR in normal breast (gray bars) and breast cancer (black bars) cell lines. Duplicate reactions were carried out for each sample, and the comparisons are for average expression.

Table III. Selected 10 novel genes showing hypermethylation in breast cancer identified by differential methylation hybridization.

\begin{tabular}{|c|c|c|c|c|c|}
\hline Gene symbol & Genebank ID & Fold of increase & Gene description & CpG site ${ }^{a}$ & eNorthern ${ }^{\mathrm{b}}$ \\
\hline NXPH1 & NM_152745 & +2.07 & Neurexophilin 1 & +1006 & $1 / 0$ \\
\hline LOC442425 & NM_001013735 & +7.82 & Forkhead box B2 & -52 & - \\
\hline NRN1 & NM_016588 & +9.3 & Neuritin 1 & +3231 & $2 / 1$ \\
\hline RAB12 & NM_001025300 & +9.2 & Member RAS oncogene family & -319 & $7 / 0$ \\
\hline CDK7 & NM_001799 & +5.7 & Cyclin-dependent kinase 7 & -195 & $13 / 3$ \\
\hline LCMT2-ADAL & NM_014793 & +4.1 & Leucine carboxyl methyltransferase 2 & -26 & $19 / 1$ \\
\hline EPSTI1 & NM_033255 & +15.1 & Epithelial stromal interaction 1 (breast) & -45 & $1 / 2$ \\
\hline CLDN1 & NM_021101 & +6.2 & Claudin 1 & -259 & $1 / 43$ \\
\hline GCLC & NM_001498 & +4.5 & Glutamate-cysteine ligase, catalytic subunit & -309 & $0 / 68$ \\
\hline CCDC4 & NM_207406 & +16.1 & Coiled-coil domain containing 4 & -96 & - \\
\hline
\end{tabular}

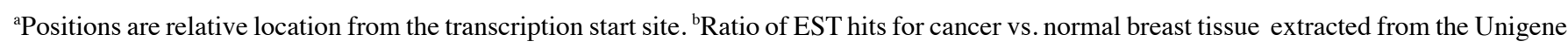
database. 
Table IV. Selected 10 novel genes showing hypomethylation in breast cancer identified by differential methylation hybridization.

\begin{tabular}{llclrr}
\hline Gene symbol & Genebank ID & Fold of decrease & \multicolumn{1}{c}{ Gene description } & CpG site $^{\mathrm{a}}$ & eNorthern $^{\mathrm{b}}$ \\
\hline ASCC3 & NM_022091 & -16.7 & Activating signal cointegrator 1 complex subunit 3 & +62 \\
HSPA4 & NM_002154 & -10.0 & Heat shock 70 kDa protein 4 & $2 / 7$ \\
NDRG3 & NM_022477 & -10.0 & NDRG family member 3 & +370 & +415 \\
BCAS3 & NM_017679 & -6.7 & Breast carcinoma amplified sequence 3 & $14 / 1$ \\
KIAA1524- & NM_020890 & -16.7 & KIAA1524 & -156 \\
DZIP3 & & & & -245 \\
FBL & NM_001436 & -5.3 & Fibrillarin & $6 / 3$ \\
PMVK & NM_006556 & -6.7 & Phosphomevalonate kinase & -51 \\
UBE2I & NM_003345 & -9.1 & Ubiquitin-conjugating enzyme E2I & $10 / 1$ \\
NXF1 & NM_006362 & -5.9 & Nuclear RNA export factor 1 & -150 \\
BAG5 & NM_004873 & -5.6 & BCL2-associated athanogene 5 & -242 \\
\hline
\end{tabular}

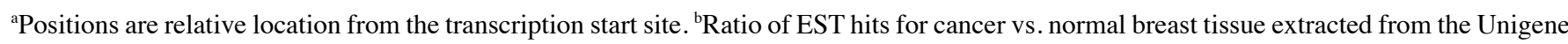
database.
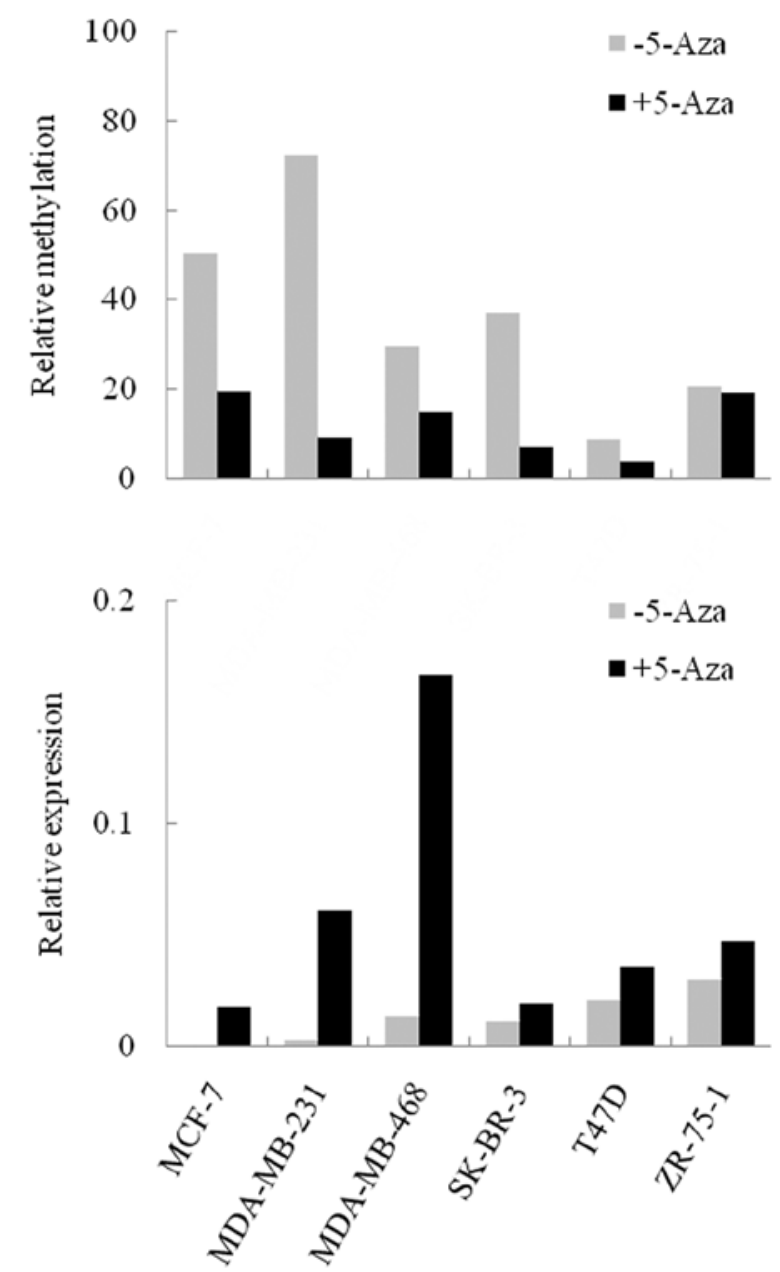

Figure 5. Induction of NRN1 expression by demethylation. (A) Induction of demethylation by 5-Aza-2'-deoxycytidine. Breast cancer cell lines were treated with 5-Aza-2'-deoxycytidine (5-Aza) and methylation level was examined by real-time MSP. Gray and black bars represent before and after treatment of 5-Aza, respectively. Each sample was examined in duplicate and the average relative methylation level is presented. (B) Recovery of expression of NRN1 after treatment of 5-Aza in breast cancer cell lines. Relative expression level of NRN1 before (gray bar) and after treatment of 5-Aza (black bar) is indicated. gene methylation was designated as 'cellular development, embryonic development, tissue development' (Fig. 6). 'Cellular movement, cancer, neurological disease (score $=36$ )' and 'cell death, embryonic development, cardiovascular disease (score = 19)' networks ranked with the next highest scores (Table V). Interestingly, STAT1, the key latent cytoplasmic transcription factor mediating various biological responses including cell proliferation, survival, apoptosis, and differentiation, features prominently in the network and appears as a master regulator of several gene transcripts that may be relevant to breast tumor development and progression (18).

Within the network, the majority of genes showed hypomethylation and only four genes showed hypermethylation. The hypermethylated genes were STAT1, TMF-1, LASP1 and NEFL1. To examine whether our tumor tissues displayed consistency between mRNA levels and DNA methylation, STAT1 and TMF-1 were chosen for RT-PCR expression analysis. STAT1 is a previously established methylation marker in a few cancer types (19). As shown in Fig. 4, both genes were down-regulated in all cancer cell lines.

The transcripts displaying the most significantly altered methylation levels within this network were MYO5A (4.56-fold decrease) and WNT3A (4.13-fold decrease). MYO5A expression was previously established as being upregulated in breast tumor tissue and may contribute to carcinogenesis and tumor progression by playing roles in the regulation of cytoskeleton, cell morphology, and filopodia motility (20). WNT3A plays a key role in $\mathrm{Wnt} / \beta$-catenin signaling and has been known to promote self-renewal of cancer stem/progenitor cells (21).

Notable cancer-related genes within the STAT1-dependent cell differentiation pathways that were hypermethylated in breast tumor included LASP1 (2.32-fold increase) and NEFL (3.42-fold increase). Significantly hypomethylated cancerrelated genes included $S M A D 7$ (2.85-fold decrease), which is a key negative regulator of TGF- $\beta$ signaling (22), and BMP4 (2.18-fold decrease) (23), which was previously found to enhance tumor growth in breast cancer. 


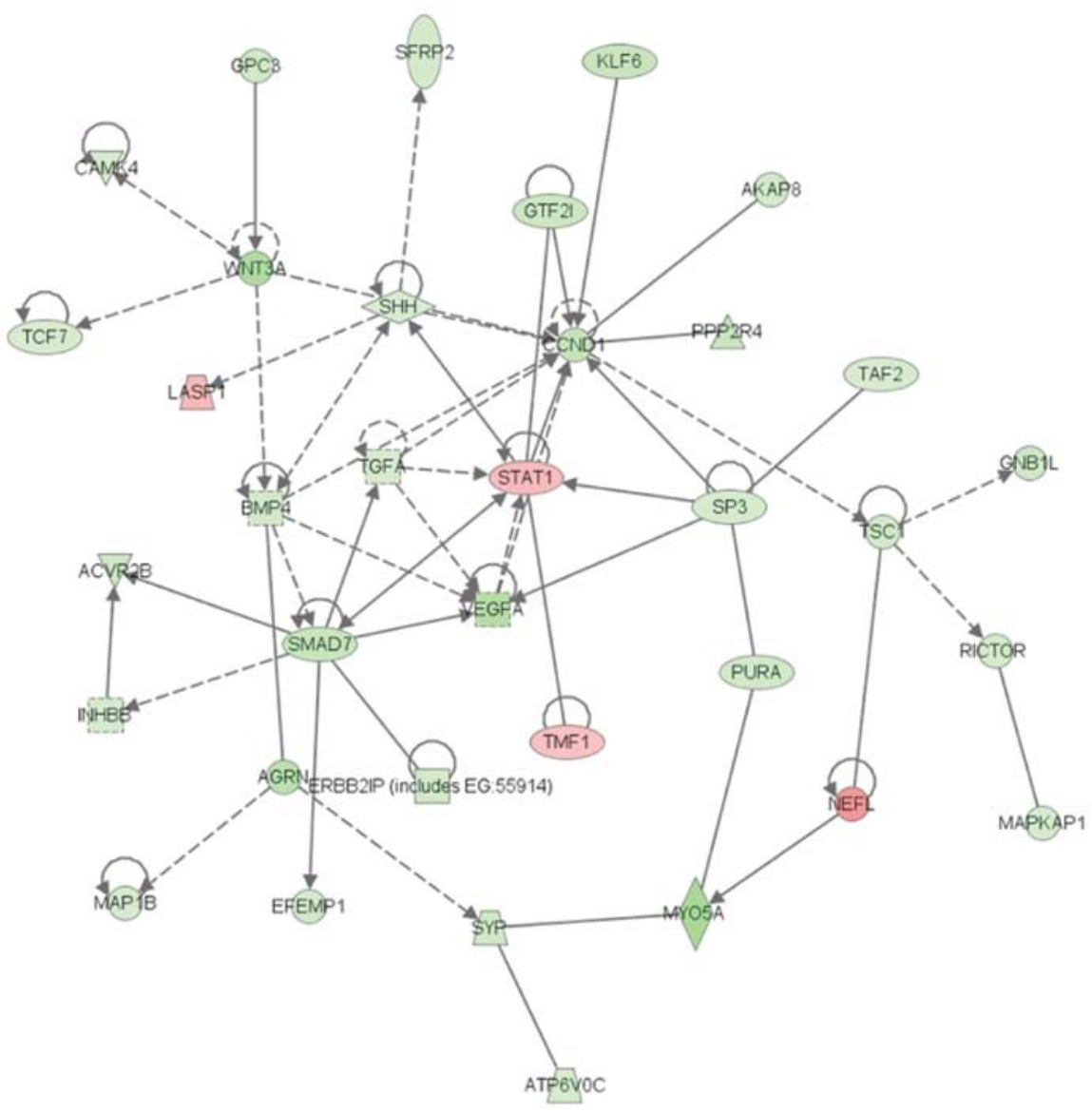

Figure 6. Highest confidence network of genes displaying altered methylation levels in breast tumor. According to IPA, the network is relevant to 'cellular development, embryonic development, and tissue development'. Genes that were hypermethylated in breast tumor are shaded in red, while those that were hypomethylated are shaded in green, with intensity signifying the magnitude of methylation change. Each interaction is supported by at least one literature reference, with solid lines representing direct interactions, and dashed lines representing indirect interactions.

Table V. Top 10 signaling pathways containing hypermethylated genes.

Cellular development, embryonic development, tissue development

Cellular movement, cancer, neurological disease

Cell death, embryonic development, cardiovascular disease

Cell signaling, nucleic acid metabolism, small molecule biochemistry

Cell death, cellular growth and proliferation, infection mechanism

Carbohydrate metabolism, lipid metabolism, small molecule biochemistry

Cellular function and maintenance, cellular compromise, cellular movement

Gene expression, genetic disorder, neurological disease

Cellular development, cellular growth and proliferation, connective tissue

Development and function

\section{Discussion}

From the analysis of the breast tumor methylation pattern, we found that hypermethylation was more prevalent than hypomethylation across the entire genome. This genome-wide hypermethylation pattern has also been observed in other cancer types including ovarian (24), prostate (25), and leukemia (26) and is therefore considered to be a general phenomenon during the course of cancer development. However, the genome-wide hypermethylation should not be confused with the global hypomethylation indicating hypomethylation of genes such as ALU and LINE that occur highly repetitive in the genome (27). Global 
hypomethylation is known to cause chromatin decondensation that results in chromosomal instability and cancer development (28). In addition, hypomethylation of repetitive elements is associated with elevated transcription, while that of normally methylated promoter $\mathrm{CpG}$ islands can lead to elevated expression of tumor antigens and possible oncogenes (29).

So far, a few genome-wide methylation analyses of breast cancer have been performed in different subtypes of cancer such as luminal/non-luminal (30) and $\mathrm{ER}^{+} / \mathrm{ER}^{-}$(15), as well as in non-specified cancer tissues (31). Most of the studies focused on specific genes such as homeobox genes (14), polycomb-binding sites (32) tumor suppressors, and oncogenes, resulting in identification of novel epigenetic markers rather than elucidating the epigenetic regulatory network involved in the carcinogenesis. GPC3, CDH2, GALNT10, and E2F7 are representative epigenetic marker genes mined by genomewide approaches. However, only a limited number of pathways are known to undergo abnormal methylation. For example, growth factor, Hun/Fos, and EGF-containing pathways were identified as the lowest $\mathrm{p}$-valued pathways in breast cancer cell lines (33). In another study by Li et al, 'Inflammatory and connective tissue disorders pathways' were identified as the most significant pathways in breast cancer (15).

Our pathway-based analysis of the biological significance of epigenetic dysregulation in breast carcinogenesis yielded a few interesting findings. Notable is the observation of altered tumor-specific methylation patterns within a network of genes whose activity may be heavily influenced by STAT1. STAT1 is a member of the signal transducer and activator of transcription factors (STAT) family: STAT1, 2, 3, 4, 5A, 5B and 6 (34). Cumulative and largely indirect evidence supports a tumor suppressor function for STAT1 $(35,36)$. Dysregulation of STAT1 signaling has been implicated in tumor formation and progression (37). The biological significance of the tumor suppressive function of STAT1 is suggested by the finding that increased expression of phosphotyrosine STAT1 in human breast cancers was associated with a statistically significant improvement in survival (38).

Genes that regulate STAT1 directly or indirectly are involved in the identified pathways. Representative genes include SMAD7, SP3, SHH, and CCND1, which interact with STAT1 directly, and TGFA, WNT3A, and VEGFA, which interact indirectly. Expression of VEGFA (39), SP3 (40), and SMAD7 (41) were known to be upregulated in breast cancer, which was consistent with our pathway analysis in which they were hypomethylated and possibly able to induce upregulation.

A key limitation of our study design is the inability to assess methylation levels with respect to tumor grade, thus precluding an early examination of potential methylation patterns specific to cancer initiation versus those specific to tumor progression. Also, detecting the frequency of methylation in tumor samples was limited due to the use of pooled DNA samples. In addition, even though there can be multiple $\mathrm{CpG}$ sites in a single gene spanning from promoter to gene-coding region, only a limited number of $\mathrm{CpG}$ were used for the pathway analysis. Nonetheless, the degree of consistency between our findings and prior reports on the sampling of cancer-related genes supports the potential of using tumor-specific alterations in methylation pattern as a prognostic tool in breast cancer development and progression.
In summary, we present pathways that were compiled using a genome-wide methylation profile of breast cancer. We found that abnormal methylation in the STAT1-involved pathways may be important in breast carcinogenesis. Further studies on the mechanisms leading to differential methylation in these regions may provide insights into the association of methylation status with tumor stage and progression that could be useful in estimating prognosis and determining treatment options for breast cancer.

\section{Acknowledgements}

This research was supported by the Basic Science Research Program through the National Research Foundation of Korea (NRF) funded by the Ministry of Education, Science and Technology (2010-0011481). Dr Kang was supported by a grant provided by the National Cancer Center, Korea.

\section{References}

1. Liu B, Tahk S, Yee KM, Fan G and Shuai K: The ligase PIAS1 restricts natural regulatory $\mathrm{T}$ cell differentiation by epigenetic repression. Science 330: 521-525, 2010.

2. Maegawa S, Hinkal G, Kim HS, et al: Widespread and tissue specific age-related DNA methylation changes in mice. Genome Res 20: 332-340, 2010.

3. Ai L, Kim WJ, Kim TY, et al: Epigenetic silencing of the tumor suppressor cystatin $\mathrm{M}$ occurs during breast cancer progression. Cancer Res 66: 7899-7909, 2006.

4. Cao Y, Vo T, Millien G, et al: Epigenetic mechanisms modulate thyroid transcription factor 1-mediated transcription of the surfactant protein B gene. J Biol Chem 285: 2152-2164, 2010.

5. Yan PS, Shi H, Rahmatpanah F, et al: Differential distribution of DNA methylation within the RASSF1A CpG island in breast cancer. Cancer Res 63: 6178-6186, 2003.

6. Matros E, Wang ZC, Lodeiro G, Miron A, Iglehart JD and Richardson AL: BRCA1 promoter methylation in sporadic breast tumors: relationship to gene expression profiles. Breast Cancer Res Treat 91: 179-186, 2005.

7. Yu J, Koujak S, Nagase S, et al: PCDH8, the human homolog of PAPC, is a candidate tumor suppressor of breast cancer. Oncogene 27: 4657-4665, 2008.

8. Wolf I, O'Kelly J, Rubinek T, et al: 15-hydroxyprostaglandin dehydrogenase is a tumor suppressor of human breast cancer. Cancer Res 66: 7818-7823, 2006.

9. Singh P, Yang M, Dai H, et al: Overexpression and hypomethylation of flap endonuclease 1 gene in breast and other cancers. Mol Cancer Res 6: 1710-1717, 2008.

10. Ito Y, Koessler T, Ibrahim A, et al: Somatically acquired hypomethylation of IGF2 in breast and colorectal cancer. Hum Mol Genet 17: 2633-2643, 2008.

11. Kim TW, Kim YJ, Lee HJ, Min SY, Kang HS and Kim SJ: Hs.137007 is a novel epigenetic marker hypermethylated and up-regulated in breast cancer. Int J Oncol 36: 1105-1111, 2010.

12. Yan PS, Perry MR, Laux DE, Asare AL, Caldwell CW and Huang TH: CpG island arrays: an application toward deciphering epigenetic signatures of breast cancer. Clin Cancer Res 6: $1432-1438,2000$.

13. Huang TH, Perry MR and Laux DE: Methylation profiling of $\mathrm{CpG}$ islands in human breast cancer cells. Hum Mol Genet 8: 459-470, 1999.

14. Tommasi S, Karm DL, Wu X, Yen Y and Pfeifer GP: Methylation of homeobox genes is a frequent and early epigenetic event in breast cancer. Breast Cancer Res 11: R14, 2009.

15. Li L, Lee KM, Han W, et al: Estrogen and progesterone receptor status affect genome-wide DNA methylation profile in breast cancer. Hum Mol Genet 19: 4273-4277, 2010.

16. Calvano SE, Xiao W, Richards DR, et al: A network-based analysis of systemic inflammation in humans. Nature 437: 1032-1037, 2005.

17. Lu L, Katsaros D, de la Longrais IA, Sochirca O and Yu H: Hypermethylation of let-7a-3 in epithelial ovarian cancer is associated with low insulin-like growth factor-II expression and favorable prognosis. Cancer Res 67: 10117-10122, 2007. 
18. Kim HS and Lee MS: STAT1 as a key modulator of cell death. Cell Signal 19: 454-465, 2007.

19. Xi S, Dyer KF, Kimak M, et al: Decreased STAT1 expression by promoter methylation in squamous cell carcinogenesis. J Natl Cancer Inst 98: 181-189, 2006.

20. Lan L, Han H, Zuo H, et al: Upregulation of myosin Va by Snail is involved in cancer cell migration and metastasis. Int J Cancer 126: 53-64, 2010.

21. Kawaguchi-Ihara N, Murohashi I, Nara N and Tohda S: Promotion of the self-renewal capacity of human acute leukemia cells by Wnt3A. Anticancer Res 28: 2701-2704, 2008.

22. Yan $X$ and Chen YG: Smad7: not only a regulator, but also a crosstalk mediator of TGF- $\beta$ signalling. Biochem J 434: 1-10, 2011

23. Alarmo EL, Kuukasjärvi T, Karhu R and Kallioniemi A: A comprehensive expression survey of bone morphogenetic proteins in breast cancer highlights the importance of BMP4 and BMP7. Breast Cancer Res Treat 103: 239-246, 2007.

24. Teschendorff AE, Menon U, Gentry-Maharaj A, et al: An epigenetic signature in peripheral blood predicts active ovarian cancer PLoS One 4: E8274, 2009.

25. Kim SJ, Kelly WK, Fu A, et al: Genome-wide methylation analysis identifies involvement of TNF- $\alpha$ mediated cancer pathways in prostate cancer. Cancer Lett 302: 47-53, 2011.

26. Deneberg S, Grövdal M, Karimi M, et al: Gene-specific and global methylation patterns predict outcome in patients with acute myeloid leukemia. Leukemia 24: 932-941, 2010.

27. Roman-Gomez J, Jimenez-Velasco A, Agirre X, et al: Repetitive DNA hypomethylation in the advanced phase of chronic myeloid leukemia. Leuk Res 32: 487-490, 2008.

28. Eden A, Gaudet F, Waghmare A and Jaenisch R: Chromosomal instability and tumors promoted by DNA hypomethylation. Science 300: 455, 2003

29. Roman-Gomez J, Jimenez-Velasco A, Agirre X, et al: Promoter hypomethylation of the LINE-1 retrotransposable elements activates sense/antisense transcription and marks the progression of chronic myeloid leukemia. Oncogene 24: 7213-7223, 2005.

30. Kamalakaran S, Varadan V, Giercksky Russnes HE, et al: DNA methylation patterns in luminal breast cancers differ from nonluminal subtypes and can identify relapse risk independent of other clinical variables. Mol Oncol 5: 77-92, 2011
31. Ruike Y, Imanaka Y, Sato F, Shimizu K and Tsujimoto G: Genome-wide analysis of aberrant methylation in human breast cancer cells using methyl-DNA immunoprecipitation combined with high-throughput sequencing. BMC Genomics 11: 137, 2010.

32. McCabe MT, Lee EK and Vertino PM: A multifactorial signature of DNA sequence and polycomb binding predicts aberrant $\mathrm{CpG}$ island methylation. Cancer Res 69: 282-291, 2009.

33. Loss LA, Sadanandam A, Durinck S, et al: Prediction of epigenetically regulated genes in breast cancer cell lines. BMC Bioinformatics 11: 305, 2010.

34. Darnell JE: STATs and gene regulation. Science 277: 1630-1635, 1997.

35. Kaplan DH, Shankaran V, Dighe AS, et al: Demonstration of an interferon gamma-dependent tumor surveillance system in immunocompetent mice. Proc Natl Acad Sci USA 95: 7556-7561, 1998.

36. Huang S, Bucana CD, Van Arsdall M and Fidler IJ: Stat1 negatively regulates angiogenesis, tumorigenicity and metastasis of tumor cells. Oncogene 21: 2504-2512, 2002.

37. $\mathrm{Yu} \mathrm{H}$ and Jove R: The STATs of cancer - new molecular targets come of age. Nat Rev Cancer 4: 97-105, 2004.

38. Widschwendter A, Tonko-Geymayer S, Welte T, Daxenbichler G, Marth C and Doppler W: Prognostic significance of signal transducer and activator of transcription 1 activation in breast cancer. Clin Cancer Res 8: 3065-3074, 2002.

39. Sharma G, Mirza S, Parshad R, et al: Clinical significance of Maspin promoter methylation and loss of its protein expression in invasive ductal breast carcinoma: correlation with VEGF-A and MTA1 expression. Tumour Biol 32: 23-32, 2011.

40. Lu S and Archer MC: Sp1 coordinately regulates de novo lipogenesis and proliferation in cancer cells. Int J Cancer 126: 416-425, 2010.

41. Papageorgis P, Lambert AW, Ozturk S, et al: Smad signaling is required to maintain epigenetic silencing during breast cancer progression. Cancer Res 70: 968-978, 2010. 